\title{
Cell rehabilitation program as a new therapeutic strategy for chronic diseases: treatment of multiple organs as a new medical hypothesis
}

\section{Editorial}

In this editorial, we put the focus on the research efforts in treating chronic diseases such as diabetes. Diabetes is known to impact various organs including brain, liver, kidneys, and reproductive system. We have also found, and others, those other organs may be subjecting to functional insufficiency or deficiency; this may lead to diabetes. Regular treatment of diabetes implies giving glucose lowering agents such as metformin and/or insulin according to the progression of the disease. From accumulating experience in research, we reached a conclusion that treatment of diabetes should be shifted to another level through what I called "cell rehabilitation, or cell reprograming". This is to say that cell can restore its normal function if its microenvironment is maintained. From a structural point of view, through examining various tissues of diabetic animals such as rats, we found that a deterioration of tissues architecture exists through the integrity of the tissue, extra spaces among the various tissue components, infiltration of inflammatory cells, and transitional changes in epithelial layers, particularly of glandular structure. We hypothesized that the involvement of neurological component may play an important role in tissue deterioration. We have continuously observed that these changes can be restored to normal circumstances in diabetic treated groups compared with diabetic groups. These observations gave evidence that chronic diseases have multifactorial agents, and that we should shift the therapeutic strategies. In other words, the body works as a machinery model rather than being restricted to a specific organ.

From our previous studies, we have found that the disease may progress if two mechanisms are working in contradicting patterns such as the up-regulation of inducible nitric oxide synthase (iNOS) in different tissues, and the downregulation of heat shock protein70 (HSP70) in the same tissues. The idea of cell rehabilitation or cell programing depends on reverse the previous events through upregulation of HSP70 and downregulation of iNOS. This programing of cells implies escaping of oxidation process and formation of free radicals that impact cells and tissues.

To prove the efficacy of this programs, we examined various herbs and found that Urtica species and Ammi visnaga can up-regulate the expression of HSP70 and downregulate the expression of iNOS. In
Volume 12 Issue 4 - 202 I

\author{
Ahed J Alkhatib 1,2 \\ 'Department of Legal Medicine, Jordan University of Science \& \\ Technology, Jordan \\ ${ }^{2}$ Department of Medicine And Critical Care, International \\ Mariinskaya Academy, USA
}

Correspondence: Ahed J Alkhatib, Department of Legal Medicine, Jordan University of Science \& Technology, Jordan, Tel 00962795905 I45, Email ajalkhatib@just.edu.jo, drahedalkatib@yahoo.com

Received: June 24, 202I | Published: July 01, 202

animal diabetic models and humans' diabetic and prediabetic models, the use of both herbs reversed the events of diabetes in terms of lowering glucose levels, HbA1c, lowering cholesterol, triglycerides, LDL, and increasing the level of HDL.

In a unique case with diabetes type 2 , a patient with glucose level of $330 \mathrm{mg} / \mathrm{dl}, \mathrm{HbAlc} 9.6 \%$, hypertension, and increased levels of cholesterol, triglycerides, LDL, and decreased levels of HDL. Following the intake of both Urtica and Ammi visnaga, for three months, there was no more diabetes, no more hypertension, and no lipid disturbances. The intake of these herbs were quitted and the levels of glucose and hypertension are normal.

Taken together, cell rehabilitation or programing deserves to be more studied as a new

\section{Acknowledgments}

None.

\section{Conflicts of interest}

The authors declare no conflicts of interest related to this article.

\section{Funding}

None. 